\title{
The Analysis of Chinese Authors' Papers in Library and Information Science
}

\author{
Chen Yuecong ${ }^{1, \text { a }}$ \\ ${ }^{1}$ Library of North China Electric Power University, Beijing 102206, China \\ acyc-79@163.com
}

Keywords: Library and Information Science; JCR; H-index

\begin{abstract}
This paper analyzed the Chinese authors' papers in Library and Information Science (LIS) from 2003 to 2014. It analyzed the Chinese papers published in journals of JCR Social Sciences Edition-- INFORMATION SCIENCE \& LIBRARY SCIENCE category. The papers' numbers and ranking in the world were listed and compared with high level countries in the world by years. Then the author's h-index, first author h-index and all papers' citations were studied.
\end{abstract}

\section{Introduction}

In recent years, more and more attention is attached to the scientific research in colleges and universities. The research of Library and Information Science (LIS) in university library has received more attention. The number of published papers in LIS has been growing rapidly in recent years.

Web of Science (previously known as (ISI) Web of Knowledge) is an online subscription-based scientific citation indexing service maintained by Thomson Reuters that provides a comprehensive citation search. It consists of SCI, SSCI, A\&HCI, CPCI-S, CPCI-SSH, and so on a series of Citation Database. It includes more than 11000 kinds of the most influential and high quality journals in the world.

Journal Citation Reports (JCR) is an annual publication by ISI in two editions. JCR Science Edition contains data about more than 8,000 journals in science and technology. JCR Social Sciences Edition contains data about more than 2,600 journals in the social sciences.

In order to understand the situation of Chinese authors in the world, JCR Social Sciences Edition is as a source of high quality papers in this paper.

\section{Research Methods and Ideas}

All the journals of the category of INFORMATION SCIENCE \& LIBRARY SCIENCE in JCR Social Sciences Edition were collected annually from 2003 to 2014. There were 55 journals in 2003, and up to 85 in 2014. All the Chinese (including Hong Kong and Taiwan, the same below) authors' papers were searched in Web of Science (WOS) and the number of papers was compared with the world's high level countries.

There were 2793 papers, among them, Chinese mainland and Hong Kong region had 1793 papers, Taiwan province had 1001 papers, and 54 papers were coauthored by Chinese mainland, Hong Kong and Taiwan. The first authors were obtained from 2793 papers and the numbers of first authors' papers were ranked from high to low. And the sample authors were selected from the high numbers of first authors. The sample authors' WOS papers, h-index, first author h-index, and all papers' citations were analyzed.

\section{Statistical Analyses of the Paper Numbers, Journals Papers Published and the Authors' Organizations}

The journals of the category of INFORMATION SCIENCE \& LIBRARY SCIENCE in JCR Social Sciences Edition were collected annually from 2003 to 2014. All the papers in the above journals were searched in Web of Science, and the region was limited to PEOPLES R CHINA (China 
mainland \& Hong Kong) and TAIWAN was not included in the data of PEOPLES R CHINA, so the TAIWAN region was analyzed alone, and the statistics were divided into with and without TAIWAN for a better comparison.

Tab.1 The paper numbers and comparisons of Chinese authors from 2003 to 2014

\begin{tabular}{|c|c|c|c|c|c|c|c|c|c|c|c|c|}
\hline & 2003 & 2004 & 2005 & 2006 & 2007 & 2008 & 2009 & 2010 & 2011 & 2012 & 2013 & 2014 \\
\hline $\begin{array}{c}\text { Number of LIS } \\
\text { journals }\end{array}$ & 55 & 54 & 55 & 53 & 56 & 61 & 66 & 77 & 83 & 85 & 84 & 85 \\
\hline $\begin{array}{c}\text { The paper number of } \\
\text { the whole world }\end{array}$ & 10286 & 9866 & 9766 & 9534 & 9446 & 9255 & 8060 & 9205 & 9475 & 8808 & 9450 & 9670 \\
\hline $\begin{array}{l}\text { China (without } \\
\text { TAIWAN) papers }\end{array}$ & 38 & 43 & 58 & 68 & 77 & 115 & 153 & 154 & 203 & 270 & 327 & 333 \\
\hline $\begin{array}{c}\text { China（without } \\
\text { TAIWAN）ranking }\end{array}$ & 11 & 9 & 6 & 6 & 7 & 5 & 5 & 5 & 6 & 3 & 3 & 3 \\
\hline $\begin{array}{c}\text { China (with } \\
\text { TAIWAN) papers }\end{array}$ & 81 & 75 & 99 & 124 & 134 & 191 & 234 & 244 & 318 & 386 & 447 & 460 \\
\hline $\begin{array}{c}\text { China (with } \\
\text { TAIWAN) ranking }\end{array}$ & 4 & 4 & 4 & 4 & 4 & 4 & 4 & 4 & 3 & 2 & 2 & 2 \\
\hline $\begin{array}{c}\text { Ranked first country } \\
\& \text { its ranking } \\
\end{array}$ & \begin{tabular}{|c|} 
USA \\
$(6,282)$ \\
\end{tabular} & \begin{tabular}{|c|} 
USA \\
$(5,672)$ \\
\end{tabular} & $\begin{array}{c}\text { USA } \\
(5,286) \\
\end{array}$ & $\begin{array}{c}\text { USA } \\
(5,443) \\
\end{array}$ & $\begin{array}{c}\text { USA } \\
(5,238) \\
\end{array}$ & $\begin{array}{c}\text { USA } \\
(5,279) \\
\end{array}$ & $\begin{array}{c}\text { USA } \\
(4,670) \\
\end{array}$ & $\begin{array}{c}\text { USA } \\
(5,291)\end{array}$ & $\begin{array}{c}\text { USA } \\
(5,309) \\
\end{array}$ & $\begin{array}{c}\text { USA } \\
(4,765)\end{array}$ & $\begin{array}{c}\text { USA } \\
(5,031)\end{array}$ & $\begin{array}{c}\text { USA } \\
(5,292)\end{array}$ \\
\hline $\begin{array}{l}\text { Ranked second/third } \\
\text { country \& its ranking }\end{array}$ & $\begin{array}{c}\text { ENGLAN } \\
\text { D (300) }\end{array}$ & \begin{tabular}{|c|} 
ENGLAN \\
D (297)
\end{tabular} & \begin{tabular}{|c|} 
CANADA \\
(281)
\end{tabular} & \begin{tabular}{|c|} 
ENGLAN \\
D (297)
\end{tabular} & \begin{tabular}{|c|} 
ENGLAN \\
D (357)
\end{tabular} & \begin{tabular}{|c} 
ENGLAN \\
D (345)
\end{tabular} & \begin{tabular}{|c} 
ENGLAN \\
D (310)
\end{tabular} & $\begin{array}{c}\text { ENGLAN } \\
\text { D (328) }\end{array}$ & $\begin{array}{c}\text { ENGLAN } \\
\text { D (354) }\end{array}$ & $\begin{array}{c}\text { ENGLAN } \\
\text { D (306) }\end{array}$ & \begin{tabular}{|c|} 
ENGLAN \\
D (337)
\end{tabular} & $\begin{array}{c}\text { ENGLAN } \\
\text { D (337) }\end{array}$ \\
\hline
\end{tabular}

Form Tab.1, there were 112821 papers all over the world, and the number of Chinese authors was no more than 100 in 2003, and increased to more than 400 in 2014. There were a lot of improvement in amount, but the number of total papers was 2793 in 12 years, while the number of United States was about five, six thousand in one year, so the gap between China and the United States is still very large.

The above 2793 papers were selected as sample papers in this paper, and there were 76 journals that the 2793 sample papers published. There were 52 kinds of journals that published more than 10 papers, and the journals with more than 200 papers were SCIENTOMETRICS (432), JOURNAL OF THE AMERICAN SOCIETY FOR INFORMATION SCIENCE AND TECHNOLOGY (215), INTERNATIONAL JOURNAL OF GEOGRAPHICAL INFORMATION SCIENCE (201) and INFORMATION \& MANAGEMENT (201).

Tab.2 The journals with more than 20 papers

\begin{tabular}{|c|c|c|c|c|c|}
\hline Ranking & Journal & Papers & Ranking & Journal & Papers \\
\hline 1 & SCIENTOMETRICS & 432 & 11 & TELECOMMUNICATIONS POLICY & 72 \\
\hline 2 & $\begin{array}{l}\text { JOURNAL OF THE AMERICAN SOCIETY FOR } \\
\text { INFORMATION SCIENCE AND TECHNOLOGY }\end{array}$ & 215 & 12 & JOURNAL OF MANAGEMENT INFORMATION SYSTEMS & 69 \\
\hline 3 & $\begin{array}{c}\text { INTERNATIONAL JOURNAL OF } \\
\text { GEOGRAPHICAL INFORMATION SCIENCE }\end{array}$ & 201 & 13 & INFORMATION SYSTEMS RESEARCH & 52 \\
\hline 3 & INFORMATION \& MANAGEMENT & 201 & 14 & GOVERNMENT INFORMATION QUARTERLY & 48 \\
\hline 5 & INFORMATION PROCESSING \& MANAGEMENT & 146 & 15 & JOURNAL OF GLOBAL INFORMATION MANAGEMENT & 46 \\
\hline 6 & ONLINE INFORMATION REVIEW & 130 & 16 & INFORMATION TECHNOLOGY \& MANAGEMENT & 45 \\
\hline 7 & ELECTRONIC LIBRARY & 124 & 17 & MIS QUARTERLY & 40 \\
\hline 8 & JOURNAL OF INFORMATION SCIENCE & 113 & 18 & JOURNAL OF ACADEMIC LIBRARIANSHIP & 32 \\
\hline 9 & $\begin{array}{c}\text { INTERNATIONAL JOURNAL OF INFORMATION } \\
\text { MANAGEMENT }\end{array}$ & 91 & 19 & $\begin{array}{l}\text { JOURNAL OF THE AMERICAN MEDICAL INFORMATICS } \\
\text { ASSOCIATION }\end{array}$ & 29 \\
\hline 10 & JOURNAL OF INFORMETRICS & 83 & 20 & LEARNED PUBLISHING & 28 \\
\hline
\end{tabular}

The first Chinese authors' organizations of 2793 sample papers were analyzed. There were 331 different organizations. Among them, City Univ Hong Kong had 152 papers and was ranked in the first. Wuhan Univ and Natl Taiwan Univ were ranked the second and third.

Tab.3 The orgnizaions with more than 30 papers

\begin{tabular}{c|c|c|c|c|c|c|c}
\hline Organizations & Papers & Organizations & Papers & Organizations & Papers & Organizations & Papers \\
\hline City Univ Hong Kong & 152 & Chinese Univ Hong Kong & 58 & Dalian Univ Technol & 47 & Inst Sci \& Tech Informat China & 35 \\
\hline
\end{tabular}




\begin{tabular}{c|c|c|c|c|c|c|c}
\hline Wuhan Univ & 145 & Fudan Univ & 58 & Natl Chengchi Univ & 47 & Natl Taiwan Univ Sci \& Technol & 34 \\
\hline Natl Taiwan Univ & 129 & Peking Univ & 57 & Nanjing Univ & 46 & Sun Yat Sen Univ & 31 \\
\hline Chinese Acad Sci & 102 & Hong Kong Univ Sci \& Technol & 54 & Natl Chung Cheng Univ & 45 & Shanghai Jiao Tong Univ & 30 \\
\hline Hong Kong Polytech Univ & 73 & Tsinghua Univ & 52 & Natl Cheng Kung Univ & 44 & \\
\hline Univ Hong Kong & 67 & Natl Cent Univ & 49 & Natl Sun Yat Sen Univ & 43 & \\
\hline Zhejiang Univ & 67 & Natl Chiao Tung Univ & 49 & Harbin Inst Technol & 42 & \\
\hline
\end{tabular}

\section{The Sample Authors` Papers in WOS and H-Index Analysis}

The first Chinese authors were extracted from 2793 sample papers, 1718 different first Chinese authors were counted after merging and duplicate removal. There were 76 authors that with more than 5 papers, and the 76 authors were selected as sample authors of this paper.

Tab4 The 76 sample authors

\begin{tabular}{|c|c|c|c|c|c|c|c|c|c|c|c|}
\hline Ranking & Author & Papers & Ranking & Author & Papers & Ranking & Author & Papers & Ranking & Author & Papers \\
\hline 1 & Huang, Mu-Hsuan & 23 & 17 & Wang, Eric T. G. & 9 & 35 & Gao, Xia & 6 & 57 & Jin, Yi & 5 \\
\hline 2 & Tsay, Ming-yueh & 17 & 17 & Wang, Yi-Shun & 9 & 35 & Ho, Yuh-Shan & 6 & 57 & Lai, Jung-Yu & 5 \\
\hline 3 & Yu, Guang & 15 & 17 & Zhang, Lin & 9 & 35 & Hong, Weiyin & 6 & 57 & Li, Aiguo & 5 \\
\hline 4 & Guan, Jiancheng & 14 & 23 & Chen, Chuanfu & 8 & 35 & Kao, Chiang & 6 & 57 & Liao, Chechen & 5 \\
\hline 5 & $\mathrm{Li}, \mathrm{Xia}$ & 13 & 23 & Xia, Jun & 8 & 35 & Leydesdorff, Loet & 6 & 57 & Lin, Tung-Ching & 5 \\
\hline 6 & $\begin{array}{c}\text { Chu, Samuel } \\
\text { Kai-Wah }\end{array}$ & 12 & 23 & Yu, Shien-Chiang & 8 & 35 & Liu, Xiaoping & 6 & 57 & Liu, Duen-Ren & 5 \\
\hline 7 & Rousseau, Ronald & 11 & 26 & Chang, Hsin Hsin & 7 & 35 & Lowry, Paul Benjamin & 6 & 57 & Liu, Xuan Zhen & 5 \\
\hline 7 & Yang, Christopher C. & 11 & 27 & Chen, Kuan-nien & 7 & 35 & Qiu, Junping & 6 & 57 & Ma, Feicheng & 5 \\
\hline 7 & \begin{tabular}{|l|} 
Ye, Fred Y. \\
\end{tabular} & 11 & 28 & Hung, Shin-Yuan & 7 & 35 & Tsai, Chih-Fong & 6 & 57 & Pei, Tao & 5 \\
\hline 7 & Zhou, Ping & 11 & 29 & Li, Jiang & 7 & 35 & Vaughan, Liwen & 6 & 57 & Ting, I-Hsien & 5 \\
\hline 11 & Davison, Robert M. & 10 & 30 & Liu, Rey-Long & 7 & 35 & Wu, Jen-Her & 6 & 57 & Tseng, Yuen-Hsien & 5 \\
\hline 11 & Hu, Xiaojun & 10 & 26 & Wu, Ing-Long & 7 & 35 & Wu, Ming-der & 6 & 57 & Wang, Weiquan & 5 \\
\hline 11 & Liang, Liming & 10 & 26 & Yu, Liangzhi & 7 & 35 & Yan, Yalan & 6 & 57 & Wang, Yuandi & 5 \\
\hline 11 & Lin, Hsiu-Fen & 10 & 26 & Zha, Xianjin & 7 & 35 & Yang, Chyan & 6 & 57 & Wei, Chih-Ping & 5 \\
\hline 11 & Liu, Yuxian & 10 & 26 & Zhang, Jin & 7 & 53 & Zhao, Rongying & 6 & 57 & Wu, Dan & 5 \\
\hline 11 & Wang, Xianwen & 10 & 35 & Chau, Michael & 6 & 54 & Cheung, Christy M. K. & 5 & 57 & Wu, I-Chin & 5 \\
\hline 17 & Chen, Yen-Liang & 9 & 35 & Chen, Dar-zen & 6 & 55 & Chuang, Kun-Yang & 5 & 57 & Xu, Yan & 5 \\
\hline 17 & Chen, Yu-Shan & 9 & 35 & Chou, Shih-Wei & 6 & 56 & Fu, Hui-Zhen & 5 & 57 & Zhao, Star X. & 5 \\
\hline 17 & Chiu, Chao-Min & 9 & 35 & Chou, Tzu-Chuan & 6 & 57 & He, Tianwei & 5 & 57 & Zhou, Tao & 5 \\
\hline
\end{tabular}

The papers of 76 sample authors in WOS were collected and the h-index of each author was counted in WOS, too.

Tab.5: The papers and h-index of 76 sample authors

\begin{tabular}{|c|c|c|c|c|c|c|c|c|c|c|c|}
\hline Author & H-index & Papers & Citations & Author & $\mathrm{H}$-index & Papers & Citations & Author & $\mathrm{H}$-index & Papers & Citations \\
\hline Leydesdorff, Loet & 32 & 197 & 3222 & Chen, Dar-zen & 10 & 89 & 313 & $\mathrm{Xu}$, Yan & 5 & 24 & 48 \\
\hline Ho, Yuh-Shan & 30 & 106 & 5056 & Vaughan, Liwen & 10 & 32 & 363 & Xia, Jun & 4 & 8 & 88 \\
\hline Guan, Jiancheng & 18 & 76 & 873 & Davison, Robert M. & 9 & 33 & 275 & Zhang, Jin & 4 & 16 & 48 \\
\hline Li, Xia & 16 & 72 & 798 & Chau, Michael & 9 & 40 & 276 & Chou, Tzu-Chuan & 4 & 21 & 99 \\
\hline Rousseau, Ronald & 16 & 135 & 1152 & Chou, Shih-Wei & 9 & 19 & 256 & Yan, Yalan & 4 & 20 & 43 \\
\hline Yang, Christopher C. & 16 & 116 & 780 & Hong, Weiyin & 9 & 13 & 359 & Wang, Yuandi & 4 & 25 & 33 \\
\hline Lin, Hsiu-Fen & 16 & 29 & 862 & Liao, Chechen & 9 & 27 & 385 & Li, Jiang & 3 & 14 & 36 \\
\hline Chen, Yen-Liang & 16 & 76 & 782 & Wei, Chih-Ping & 9 & 43 & 214 & Liu, Rey-Long & 3 & 20 & 28 \\
\hline Cheung, Christy M. K. & 16 & 55 & 939 & Zhou, Ping & 8 & 16 & 183 & Yu, Liangzhi & 3 & 9 & 52 \\
\hline Liu, Duen-Ren & 16 & 81 & 776 & Yu, Guang & 7 & 31 & 105 & Zha, Xianjin & 3 & 29 & 25 \\
\hline Chiu, Chao-Min & 15 & 26 & 1276 & Liang, Liming & 7 & 22 & 359 & Wu, Ming-der & 3 & 7 & 21 \\
\hline Wang, Eric T. G. & 14 & 35 & 1007 & Fu, Hui-Zhen & 7 & 17 & 118 & He, Tianwei & 3 & 7 & 37 \\
\hline Wang, Yi-Shun & 14 & 34 & 1090 & Lai, Jung-Yu & 7 & 19 & 85 & Li, Aiguo & 3 & 5 & 16 \\
\hline Chang, Hsin Hsin & 14 & 38 & 544 & Lin, Tung-Ching & 7 & 23 & 274 & Ma, Feicheng & 3 & 32 & 46 \\
\hline Kao, Chiang & 14 & 49 & 892 & Pei, Tao & 7 & 38 & 215 & Wu, Dan & 3 & 22 & 28 \\
\hline Tsai, Chih-Fong & 14 & 65 & 573 & Ye, Fred Y. & 6 & 38 & 155 & Wu, I-Chin & 3 & 12 & 37 \\
\hline Chen, Yu-Shan & 13 & 51 & 657 & $\mathrm{Hu}$, Xiaojun & 6 & 32 & 107 & Zhao, Star X. & 3 & 14 & 39 \\
\hline Liu, Xiaoping & 13 & 52 & 489 & Liu, Yuxian & 6 & 18 & 125 & Chen, Chuanfu & 2 & 15 & 16 \\
\hline Wu, Jen-Her & 13 & 31 & 1001 & Wang, Xianwen & 6 & 21 & 84 & Chen, Kuan-nien & 2 & 10 & 8 \\
\hline Zhou, Tao & 13 & 43 & 487 & Gao, Xia & 6 & 10 & 83 & Jin, Yi & 2 & 5 & 16 \\
\hline Hung, Shin-Yuan & 12 & 37 & 512 & Chuang, Kun-Yang & 6 & 15 & 166 & Liu, Xuan Zhen & 2 & 6 & 21 \\
\hline Lowry, Paul Benjamin & 12 & 44 & 433 & Tseng, Yuen-Hsien & 6 & 20 & 231 & Yu, Shien-Chiang & 1 & 5 & 5 \\
\hline Huang, Mu-Hsuan & 11 & 75 & 339 & Tsay, Ming-yueh & 5 & 22 & 105 & Zhao, Rongying & 1 & 14 & 15 \\
\hline Yang, Chyan & 11 & 60 & 347 & Chu, Samuel Kai-Wah & 5 & 20 & 87 & Ting, I-Hsien & 1 & 19 & 7 \\
\hline Zhang, Lin & 10 & 47 & 306 & Qiu, Junping & 5 & 31 & 61 & & & & \\
\hline Wu, Ing-Long & 10 & 27 & 353 & Wang, Weiquan & 5 & 8 & 175 & & & & \\
\hline
\end{tabular}


The papers of 76 sample authors in WOS were analyzed and not first author papers were excluded. Then the papers of first authors were left, and the first author h-indexes were counted as corresponding.

Tab.6 The comparison of first and not first author

\begin{tabular}{|c|c|c|c|c|c|c|c|c|c|c|c|}
\hline Author & Papers & Papers' & $H$-index & $H$-index' & Decrease in \% & Author & Papers & Papers' & $H$-index & H-index & $\begin{array}{c}\text { Decrease } \\
\text { in } \%\end{array}$ \\
\hline Leydesdorff, Loet & 197 & 105 & 32 & 27 & $-15.63 \%$ & Wu, Jen-Her & 31 & 21 & 13 & 12 & $-7.69 \%$ \\
\hline Ho, Yuh-Shan & 106 & 64 & 30 & 21 & $-30.00 \%$ & Liu, Duen-Ren & 81 & 41 & 16 & 12 & $-25.00 \%$ \\
\hline Lin, Hsiu-Fen & 29 & 28 & 16 & 16 & $0.00 \%$ & Zhou, Tao & 43 & 41 & 13 & 12 & $-7.69 \%$ \\
\hline Guan, Jiancheng & 76 & 40 & 18 & 15 & $-16.67 \%$ & Yang, Christopher C. & 116 & 53 & 16 & 11 & $-31.25 \%$ \\
\hline Chen, Yen-Liang & 76 & 47 & 16 & 14 & $-12.50 \%$ & Chiu, Chao-Min & 26 & 18 & 15 & 11 & $-26.67 \%$ \\
\hline Chang, Hsin Hsin & 38 & 37 & 14 & 14 & $0.00 \%$ & $\mathrm{Li}, \mathrm{Xia}$ & 72 & 17 & 16 & 10 & $-37.50 \%$ \\
\hline Kao, Chiang & 49 & 43 & 14 & 14 & $0.00 \%$ & Wang, Yi-Shun & 34 & 20 & 14 & 10 & $-28.57 \%$ \\
\hline Tsai, Chih-Fong & 65 & 40 & 14 & 13 & $-7.14 \%$ & Hung, Shin-Yuan & 37 & 27 & 12 & 10 & $-16.67 \%$ \\
\hline Chen, Yu-Shan & 51 & 43 & 13 & 12 & $-7.69 \%$ & Wu, Ing-Long & 27 & 25 & 10 & 10 & $0.00 \%$ \\
\hline Wang, Eric T. G. & 35 & 19 & 14 & 12 & $-14.29 \%$ & & & & & & \\
\hline
\end{tabular}

The first author h-index more than 10 was listed in Tab.6 with 19 authors. The papers of Lin, Hsiu-Fen;Chang, Hsin Hsin; Kao, Chiang and Wu, Ing-Long are all first author papers, so the h-indexes' were not decreased in these four authors. But the difference of papers and first author papers of Ho, Yuh-Shan; Yang, Christopher $\mathrm{C}$ and $\mathrm{Li}$, Xia were more than the others, so the h-indexes' decreased significantly, about 30\%. The h-index' of other 12 authors also decreased in varying degrees.

\section{The Sample Papers Citation Analysis in WOS}

There were 2227 papers in 2793 papers that cited by papers in WOS. The papers' citation times more than 100 times were listed in Tab.7. There were 29 papers in Tab.7, in which the first Chinese authors were all from Hong Kong or Taiwan. This showed that the high cited papers in the sample papers were all from Hong Kong or Taiwan.

Tab.7 The citation times more than 100

\begin{tabular}{|c|c|c|c|c|c|c|c|}
\hline $\begin{array}{c}\text { Autho } \\
r\end{array}$ & Citaions & $\begin{array}{c}\text { The first Chinese } \\
\text { author }\end{array}$ & Organization & Author & Citaions & The first Chinese author & Organization \\
\hline 1 & 707 & Lee, JN & City Univ Hong Kong & 16 & 142 & Tseng, Yuen-Hsien & Natl Taiwan Normal Univ \\
\hline 2 & 529 & Wei, KK & City Univ Hong Kong & 17 & 136 & Shang, Rong-An & Soochow Univ \\
\hline 3 & 473 & Ho, Yuh-Shan & Taipei Med Univ & 18 & 135 & Chow, Wing S & Hong Kong Baptist Univ \\
\hline 4 & 361 & Wu, Jen-Her & Natl Sun Yat Sen Univ & 19 & 133 & Sher, PJ & Natl Chung Hsing Univ \\
\hline 5 & 344 & $\mathrm{Hsu}, \mathrm{CL}$ & Natl TAiwan Univ Sci \& Technol & 20 & 131 & Yang, ZL & City Univ Hong Kong \\
\hline 6 & 286 & Hsu, Chin-Lung & Da Yeh Univ & 21 & 130 & Lin, Hsin-Hui & Natl Taiwan Inst Technol \\
\hline 7 & 207 & Thong, James Y. L. & City Univ Hong Kong & 22 & 128 & Lee, Jae-Nam & City Univ Hong Kong \\
\hline 8 & 179 & Thong, James Y. L. & Hong Kong Univ Sci \& Technol & 23 & 128 & Wu, Jen-Her & Natl Sun Yat Sen Univ \\
\hline 9 & 178 & Lee, MKO & City Univ Hong Kong & 24 & 127 & Xu, Sean Xin & Hong Kong Univ Sci \& Technol \\
\hline 10 & 170 & lin,Cathy S. & Natl Univ Kaohsiung & 25 & 121 & Theodore H.K. Clark & Hong Kong Univ Sci \& Technol \\
\hline 11 & 162 & Lin, Hsiu-Fen & Natl Taiwan Ocean Univ & 26 & 117 & Davison, Robert M. & City Univ Hong Kong \\
\hline 12 & 158 & Ong, Chorng-Shyong & Natl Taiwan Univ & 27 & 115 & Hsieh, J. J. Po-A & Hong Kong Polytech Univ \\
\hline 13 & 154 & Shih, Hung-Pin & Hsuan Chuang Univ & 28 & 103 & Thong, James Y. L. & Hong Kong Univ Sci \& Technol \\
\hline 14 & 146 & Wang, Yi-Shun & Natl Changhua Univ Educ & 29 & 102 & Tam, Kar Yan & Hong Kong Univ Sci \& Technol \\
\hline 15 & 145 & Hong, Se-Joon & Hong Kong Univ Sci \& Technol & & & & \\
\hline
\end{tabular}

\section{Conclusions}

The Chinese authors' papers in Library and Information Science (LIS) from 2003 to 2014 were analyzed in this paper. The papers of Chinese authors in the category INFORMATION SCIENCE \& LIBRARY SCIENCE of JCR were increasing rapidly from 2003 to 2014, but there was still a large gap compared with USA. The h-index of the sample authors were discussed, and the highest h-index was 32 and the author was Leydesdorff, Loet, H from Univ Amsterdam, his first author paper was 105. The first author h-index was related with the author's first author papers, and it decreased a lot if most of his papers were not first author papers. Otherwise, if the authors' papers were all first author papers, the h-index was equal with the first author h-index of him. Most of the 2793 papers were cited in WOS, and the first Chinese author of high cited papers were all from Hong Kong or Taiwan. 


\section{References}

[1] LIU Rui-yuan; LIU Xue-li; WANG Pu; ZHENG Cheng-ming. International Status of Chinese Library Science and information Science: Literature Metrology Analysis and Evaluation Based on the Database of the SSCI [J]. Information Science, 2014, 04:101-105.

[2] CHE Yao; PAN Yun-tao; ZHAO Xiao-yuan; YAO Chang-qing. 2012 Annual Research Evolution of Information Science_- Thesis Statistics Analysis of International Library and Information Science Core Journals in which Chinese Scholar had been published [J]. Information Science, 2014, 09:155-161.

[3] FENG Zhu-bin; HUA Wei-na. Scientometric Study on Library and Information Science Research Productivity in China Based on Web of Science [J]. Document, Information \& Knowledge, 2013, 04:42-50.

[4] MAO Yi-guo; CHEN Jian-guang. The Internalization Trend of Library and Information Science in China [J]. Library Tribune, 2014, 05:8-14.

[5] QIU Jun-ping; Zeng, Qian. Study on Chinese Library and Information Science International Papers [J]. Library \& Information, 2013, 01:90-97. 